\title{
Design of a miniature electrolyte conductivity probe using ISFETs in a four point configuration
}

\author{
A. Volanschi*, W. Olthuis and P. Bergveld \\ MESA Research Institute, University of Twente, PO Bax 217, 7500 AE Enschede (Netherlands)
}

\begin{abstract}
In this work the design, fabrication and testing of a four point electrode configuration based on ISFETs for the measurement of electrolytic conductivity are presented. The final design uses a distance between the ISFET gates of only $100 \mathrm{~mm}$ allowing a chip area in contact with the electrolyte of $2.2 \times 2.2 \mathrm{~mm}$. KCl electrolyte concentrations ranging from $0.1 \mathrm{mM}$ to $1 \mathrm{M}$ were used for testing the designed probe. Measurement results show a linear dependence of the output voltage of the amplifier on the electrolyte concentration.
\end{abstract}

\section{Introduction}

Electrolyte conductance measurements can be carried out easily by means of commercially available, dedicated equipment. However, research effort is still being invested in the field of integrated chemical sensors, e.g. towards the integration of several measurement functions in only one probe.

This work proposes a measurement method for electrolyte conductivity using ISFETs. The ISFET, a solid state device originally designed for $\mathrm{pH}$ measurements [1], can also be used for conductivity measurements in electrolytes [2] and in membranes containing charged proteins [3]. It means that two measurement functions, i.e. $\mathrm{pH}$ and conductivity, could possibly be integrated into one probe.

The method is, basically, an application of the wellknown four point measurement principle. According to this principle, current is applied through two electrodes and voltage is measured with two other electrodes placed in-between the former electrodes. The current produces an electric field in the electrolyte and the resulting potential difference is measured. ISFETs can be used for this voltage measurement as they can operate as potential sensing electrodes besides their $\mathrm{pH}$ sensing properties.

\section{Design and fabrication}

Conventionally, two inert metal electrodes (e.g. platinum), immersed in the electrolyte solution, are used

*Author to whom correspondence should be addressed. for measuring the electrolyte conductivity. Figure 1 shows the equivalent circuit of a two-electrode planar structure. The impedance, $Z$, of the structure can be determined by applying an a.c. current and measuring the resulting voltage. The electrolyte conductivity, $\sigma(\mathrm{mS} /$ $\mathrm{cm})$, is related to the electrolyte resistance, $R_{\mathrm{el}}(\mathrm{k} \Omega)$ through

$R_{\mathrm{el}}=\frac{1}{\sigma} K_{\mathrm{c}}$

where $K_{\mathrm{c}}$ is the cell constant $\left(\mathrm{cm}^{-1}\right)$. However, the measured impedance, $Z$, and the electrolyte resistance, $R_{\mathrm{el}}$, to be determined are not identical, unless the other components from the scheme in Fig. $1\left(C_{\mathrm{par}}, Z_{\mathrm{t}} C_{\mathrm{d} \text {, }}\right.$ etc.) can be ruled out.

Several methods to make $R_{\mathrm{el}}$ prevail are described in the literature [4-6]. The most common solution is platinisation [5]. The effective electrode surface is increased and the impedance corresponding to $C_{\mathrm{d}}$ is decreased. The results are acceptable if the initial

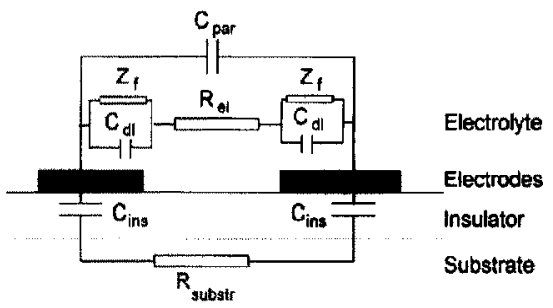

Fig. 1. Equivalent circuit of a two-electrode planar structure immersed in electrolyte. $c_{p m i}$ represents the parasitic capacitance, $Z_{f}$ the Faradaic impedance, $C_{\mathrm{df}}$ the double layer capacitance, $R_{\mathrm{d}}$ the electrolyte resistance, $C_{\text {ins }}$ the capacitance of the insulator, and $R_{\text {substs }}$ the substrate resistance. 
electrode area is not too small (in the order of $1 \mathrm{~cm}^{2}$ ). The method is not suited for probe miniaturisation. Another way to reduce the electrode impedance is to use a planar interdigitated array. Although a miniature probe can be made, this method has the disadvantage that the parasitic capacitance of the structure $\left(C_{\mathrm{par}}\right)$ is larger [6]. A third way to control the influence of the electrode impedance is to cover the electrode surface with a special insulator layer [4]. Consequently, the electrode impedance is fixed to a reproducible value, independent of the electrolyte concentration. This impedance can than be ruled out by signal processing. However, the resulting probe is not very small.

The electrode impedance can also be completely separated from $R_{\mathrm{el}}$ and thus from the conductivity, $\sigma$, if a four-electrode configuration is used, two electrodes for injection of the current and two electrodes for the measurement of the potential difference. The advantage of this approach is that the impedance of the current injection electrodes is not significant for the conductivity measurement. Consequently, these electrodes can be fabricated very small and the probe as a whole can be miniaturised.

Considering the advantages and disadvantages of the methods mentioned above, the preferred choice is the four point electrode configuration. The idea is to use ISFETs as the potential sensing electrodes placed inbetween the current injection electrodes.

\section{Probe layout}

A cross section of the probe, showing the two ISFETs placed in-between two metal electrodes is presented in Fig. 2. Also shown in Fig. 2 are the most important layout parameters. The design of the layout is based on a series of conditions.

The resistance of the electrolyte in-between the ISFET gates and the current injected through the current electrodes determine the voltage difference between the gates. This voltage difference should be always at least ten times larger than the noise of the transistors caused by stochastic chemical and electrical phenomena (about $10 \mu \mathrm{V}$ ). Furthermore, the measured resistance between the ISFET gates is a function of the distance between the gates, $a$.

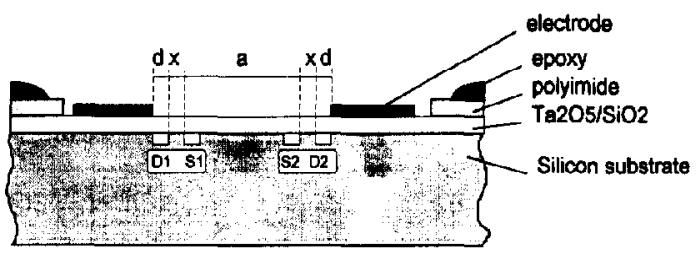

Fig. 2. Cross section of an actual four point conductivity probe based on ISFETs. $a=100 \mu \mathrm{m}, x=15 \mu \mathrm{m}, d=15 \mu \mathrm{m}$. The electrode size is $1 \times 2 \mathrm{~mm}$. Electrodes are made from platinum.
Estimation of the voltage difference between the gates can be made on a simplified two-electrode planar structure like the one shown in Fig. 1. Based on the available area on a usual ISFFT chip $(3 \times 4 \mathrm{~mm})$, the electrode area used for this estimation was $2 \times 1 \mathrm{~mm}$. Two distances between the edges of the electrodes have been considered, 110 and $160 \mu \mathrm{m}$. The calculated cell constants were 3.86 and $4.32 \mathrm{~cm}^{-1}$, respectively. The calculations were done with the program Trendy, a process and device simulation program for development of semiconductor devices $[7,8]$. An estimation of the voltage between the electrodes can be done now according to eqn. (2) (cf. eqn. (1)):

$V=\frac{1}{\sigma} K_{\mathrm{c}} I$

where $I$ is the current injected through the electrodes. For a current of $0.5 \mu \mathrm{A}$ and an electrolyte conductivity of $1 \mathrm{mS} / \mathrm{cm}$ (corresponding to an $8 \mathrm{mM} \mathrm{KCl}$ solution) the resulting voltage between the electrodes is 1.93 and $2.16 \mathrm{mV}$, respectively. The same current but a conductivity of $22 \mathrm{mS} / \mathrm{cm}$ (corresponding to a $0.2 \mathrm{M} \mathrm{KCl}$ solution) gives $0.098 \mathrm{mV}$ for the structure with a 160 $\mu \mathrm{m}$ distance between the electrodes. If the distance between the ISFET gates, $a$, is not made much smaller than $160 \mu \mathrm{m}$, a voltage difference of the same order of magnitude is expected between the gates, i.e. a signal that is high enough in comparison with the mentioned transistor noise.

The available technology of the ISFET [1] uses a standard gate length, $x$, of $15 \mu \mathrm{m}$. Because the distance between the electrode and the ISFET gate, $d$, is not representative for the conductivity measurement and because of the noise considerations where a maximal distance $a$ between the ISFET gates was preferred, it is indicated to keep the distance, $d$, as small as possible. The distance $d$ was chosen to be $15 \mu \mathrm{m}$. The resulting distance $a$ between the gates is $(160-4 \times 15) \mu \mathrm{m}=100$ $\mu \mathrm{m}$.

To deposit and pattern platinum electrodes on both sides of the gates, additional steps were introduced in the mentioned ISFET fabrication process [1]. The metal electrodes consist of a sandwich of sputtered titanium and platinum layers with a thickness of 50 and 250 $\mathrm{nm}$, respectively. The electrode were patterned by a lift-off process.

Finally, the chip area in contact with the electrolyte was defined by a polyimide window $(2.2 \times 2.2 \mathrm{~mm})$ outside the electrode area. The probes were further mounted on $8 \times 100 \mathrm{~mm}$ printed circuit board carriers and encapsulated in epoxy.

\section{Circuitry}

To measure the difference of the potential on the ISFET gates a differential amplifier was designed. A 




Fig. 3. Simplified scheme of the differential amplifier used for testing the electrolyte conductivity measurement probe.

simplified scheme of the circuit used is given in Fig. 3. The amplifier was designed with the operational amplifier AD 841JN (Analog Devices). A Philips broad bandwidth millivoltmeter (model PM 2554) was used to read the output voltage of the amplifier. The ISFET d.c. current, $I_{\mathrm{ss}}$ in Fig. 3, was $1.55 \mathrm{~mA}$. The current source was simulated with a function generator (Siemens, model D2011-8AA) in series with a resistor with high resistance, much higher than the impedance of the current injection electrode system. The frequency, the current and the internal resistance of the source can in this way be changed easily. A resistance of 4.3 $\mathrm{k} \Omega$ of the electrolyte in-between the current injection electrodes was calculated assuming an electrolyte conductivity of $1 \mathrm{mS} / \mathrm{cm}$. The value of the resistors used for the current source were chosen much higher than this calculated value, i.e. 1 or $10 \mathrm{M} \Omega$, resulting in an injected current of 0.56 and $5.6 \mu \mathrm{A}$ r.m.s., respectively.

It is expected that no reference electrode is needed for the conductivity measurement. Although the platinum-electrolyte interface is thermodynamically undefined, and thus generates an unstable voltage, this voltage is measured by the differential ISFET pair as common mode voltage and will therefore not result in an amplifier output voltage [9].

The upper frequency limit of the current is determined by the necessity to reduce the loading of the current source by the parasitic capacitance of the probe and of the connection wires. The lower frequency limit is not critical. Considering the fact that replacing one of the ISFETs with a REFET would make a $\mathrm{pH}$ measurement possible [9], it is preferred to set the working frequency of the conductivity measurement higher than the cut-off frequency of the amplifier of the $\mathrm{pH}$-meter. Conclusively, a frequency of $1 \mathrm{kHz}$ was chosen for the conductivity measurements.

\section{Results and discussion}

Measurements in $\mathrm{KCl}$ solutions with various concentrations ranging from $0.1 \mathrm{mM}$ to $1 \mathrm{M}$, at room

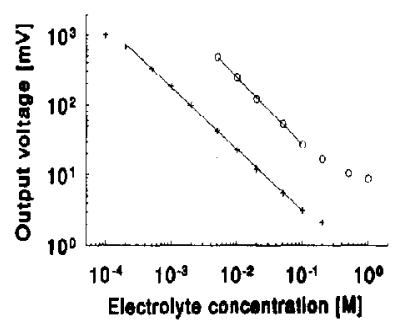

Fig. 4. Output voltage of the differential amplifier ( $V_{\text {out }}$ in Fig. 3) as a function of the electrolyte concentration $(\mathrm{KCl})$. The current injected through the electrodes is $0.56(+)$ and $5.6(0)$ $\mu \mathrm{A} \mathrm{r.m.s}$. The frequency of the signal is $1 \mathrm{kHz}$.

temperature $\left(21 \pm 2^{\circ} \mathrm{C}\right)$, were done to test the designed electrolyte conductivity probe and the corresponding circuitry. Results are presented in Fig. 4. The output voltage of the differential amplifier varies linearly with the conductivity of the $\mathrm{KCl}$ solution over three decades (for the $0.56 \mu \mathrm{A}$ current source). Departure from linearity at high electrolyte concentration can be explained through the effect of the reduced activity of the potassium and chlorine ions on the electrolyte conductivity. Even at $\mathrm{KCl}$ concentrations as high as 0.2 $M$ that have been used for the simulations, the noise of the transistors did not disturb the measurements. The results obtained confirm the fact that no reference electrode is needed.

To change the measured conductivity range according to a specific application, one should change the distance between the ISFET gates. Reducing the distance results in a shift of the measurement range towards lower electrolyte conductivity. In contrast, an increase in the distance $a$ between the gates should lead to a shift of the measurement range towards higher electrolyte conductivity. Smaller shifts of this conductivity range can be achieved by adjusting the current injected through the metal electrodes.

\section{Conclusions}

It is shown in this paper that the ISFET cannot only be used for $\mathrm{pH}$ measurements, but also for potential sensing. Based on this property, an electrolyte conductivity measurement probe using ISFETs in a four point electrode configuration was designed, fabricated and tested. More than three electrolyte conductivity decades have been covered, without any correction in the electronic circuitry.

The chosen electrode arrangement made it possible to miniaturise the conductivity cell. The conductivity probe uses a distance of $100 \mu \mathrm{m}$ between the gates of the ISFETs. The final chip area in contact with the electrolyte is $2.2 \times 2.2 \mathrm{~mm}$. Because a differential con- 
figuration of the ISFETs is used, the way remains open for the integration of a $\mathrm{pH}$ sensor and a conductivity cell in one small device, if one of the ISFETs is replaced by a REFET.

\section{References}

1 P. Bergueld and A. Sibbald, in G. Svehla (ed.), Comprehensive Analytical Chemistry, Vol. XXIII, Elsevier, Amsterdam, 1988.

2 A. Volanschi, W. Olthuis and P. Bergveld, Electrolyte conductivity measured with an ISFET, Proc. Conf. Trends in Electrochemical Biosensors, Trieste, Italy, June 1992

3 J. Kruise, J.G. Rispens, P. Bergueld, F.J.B. Kremer, D.A.J. Starmans, J.R. Haak, J. Feijen and D.N. Reinhoudt, Detection of charged proteins by means of impedance measurements, Sensors and Actuators B, 6 (1992) 101-105.
4 W. Olthuis, A. Volanschi, J.G. Bomer and P. Bergveld, A new probe for measuring electrolyte conductance, Sensors and Actuators B, 13-14 (1993) 230-233.

$5 \mathrm{G}$. Jones and D. Bollinger, The measurement of the conductance of electrolytes; on platinisation, J. Am. Chem. Soc., 57 (1935) 280-284.

6 R.Y. Scapple, A trimmable planar capacitor for hybrid applications, Proc. 24th Electronic Components Conf., New York, $U S A, 1974$, pp. 203-207.

7 E. van Schie, Trendy, an integrated program for IC process and device simulation, Thesis, University of Twente, Netherlands, 1990.

8 P.B.M. Wolbert, Modeling and simulation of semiconductor devices in Trendy, Electrical, thermal and hydrodynamic behaviour, Thesis, University of Twente, Netherlands, 1991.

9 P.Bergveld, A.van den Berg, P.D. van der Wal,M. SkowronskaPtasinska, E.J.R. Sudhölter and D.N. Reinhoudt, How electrical and chemical requirements for REFETs may coincide, Sensors and Actuators, 18 (1989) 309-327. 